\title{
Revealing the Structure of Graphitic Carbon Nitride through Low-Dose TEM using a Direct Electron Detector
}

\author{
Diane M. Haiber ${ }^{1}$ and Peter A. Crozier ${ }^{1}$ \\ 1. School for Engineering of Matter, Transport \& Energy, Arizona State University, Tempe, Arizona, \\ USA
}

Graphitic carbon nitrides (commonly referred to as $\mathrm{g}-\mathrm{CN}_{\mathrm{x}} \mathrm{H}_{\mathrm{y}}$ or $\mathrm{g}-\mathrm{C}_{3} \mathrm{~N}_{4}$ ) have recently garnered interest due to their visible-light activity for photocatalytic water reduction [1]. Similar to graphite, g- $\mathrm{CN}_{\mathrm{x}} \mathrm{H}_{\mathrm{y}}$ has a hexagonally symmetric in-plane structure but with periodic replacement of $\mathrm{C}$-atoms with $\mathrm{N}$-atoms resulting in bandgap formation and regular voids within the layers. Due to the synthesis strategy, residual hydrogen becomes trapped in the structure thus preventing long range planar order [2]. Because g- $\mathrm{CN}_{\mathrm{x}} \mathrm{H}_{\mathrm{y}}$ is extremely beam sensitive, conventional high dose imaging with transmission electron microscopy (TEM) renders the material amorphous [3]. However, by combining low electron doses with direct electron detectors, the structure of $\mathrm{g}-\mathrm{CN}_{\mathrm{x}} \mathrm{H}_{\mathrm{y}}$ can be imaged using high-resolution TEM.

All TEM images were collected on an image-corrected FEI Titan electron microscope operating at 300 $\mathrm{kV}$. To enhance the bright contrast of atoms/motifs, a negative spherical aberration coefficient of $-20 \mu \mathrm{m}$ was employed. All images were acquired on a Gatan K2 IS detector operated in counting mode. As such, the Titan monochromator focus was adjusted to achieve a dose between $\sim 4-7 \mathrm{e} / \mathrm{pixel} / \mathrm{s}$ on the detector array. A commercial $\mathrm{g}-\mathrm{CN}_{\mathrm{x}} \mathrm{H}_{\mathrm{y}}$ powder was obtained from Carbodeon Ltd. and used as-received. Preparation of a TEM specimen involved ultrasonicating an aqueous suspension of the crushed powder for 12 hours followed by submerging a lacey- $\mathrm{C} / \mathrm{Cu}$ grid in the solution and allowing it to air dry. To interpret the TEM images, an X-ray diffraction (XRD) pattern was collected on the bulk powder.

Two representative images of $\mathrm{g}-\mathrm{CN}_{\mathrm{x}} \mathrm{H}_{\mathrm{y}}$, including the pseudo-(001) zone axis and a side view of the graphitic layers, are shown in Figure 1(a)-(b). To achieve a good signal-to-noise ratio in the plan-view image, a 10-second total exposure was broken into 100 frames and alignment of the stack was performed using a bandpass filter in GMS-3 software. From the FFT of the plan-view image (Fig. l(c)), the crystallinity of this material becomes apparent with the most prominent spatial frequency corresponding to the in-plane repeat distance separating heptazine motifs at $\sim 6.7 \AA$. Diffuse intensity and broken rings in the FFT suggest the layer stacking may be randomly oriented. Fig. 1( $a$ ) also appears to consists of multiple sheets and buckled regions, thus reducing the ability to distinguish the 2D-motif. The corresponding FFT of the side-view image (Fig.1(e)) reveals the "graphitic" separation distance of $3.2 \AA$. Figure $1(d)$ contains the XRD pattern for this material and the proposed structure model of a fully-condensed $\mathrm{g}-\mathrm{C}_{3} \mathrm{~N}_{4}$ single sheet, both of which were used to index the FFT's and qualitatively interpret the image contrast, respectively. Fourier analysis was performed on the boxed region in the plan-view image, Fig.2(a), to quantitatively interpret some of the image contrast. Figure 2(b) contains the FFT of the region of interest showing six well-defined central spots corresponding to an average spacing of $6.4 \AA$. After isolating these (100)-like spots using a mask tool (Fig.2(c)), an inverse-FFT (IFFT) was performed to yield an image containing contrast from only the bridged-heptazine units (Fig.2(d)). The region of interest together with the IFFT (Fig.2(e)) clearly illustrate that the bright and dark regions are indeed due to the alternating heptazine/void in-plane structure of condensed g- $\mathrm{C}_{3} \mathrm{~N}_{4}$, on the nanometer length scale. Real-space averaging techniques will be combined with higher resolution imaging to explore the extent of planar and stacking disorder in $\mathrm{g}-\mathrm{CN}_{\mathrm{x}} \mathrm{H}_{\mathrm{y}}$ materials [4]. 
References:

[1] X. Wang et al. Nat. Mater. 8, (2009), p. 76.

[2] B.V. Lotsch et al. Chem. Eur. J. 13, (2007), p. 4969.

[3] D.M. Haiber et al. Microsc. Microanal. 22 (Suppl 3), (2016), p. 986.

[4] Support from the U.S. DOE (DE-SC0004954), use of AC-TEM at the John M. Cowley Center for High Resolution Electron Microscopy, use of the K2 IS camera courtesy of Gatan, and use of XRD equipment within the LeRoy Eyring Center for Solid State Science at ASU are gratefully acknowledged.
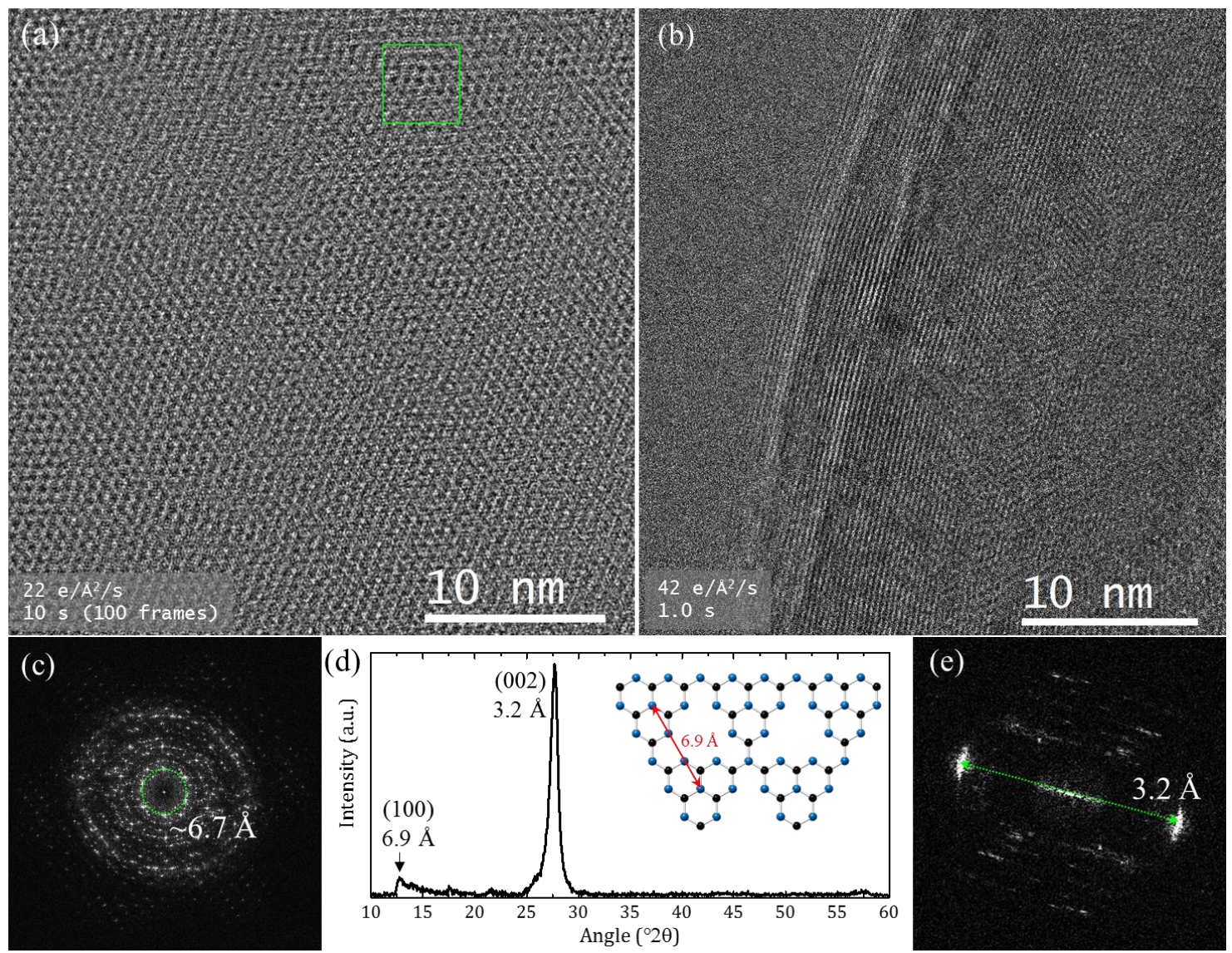

Figure 1. Low-dose TEM images of $g-\mathrm{CN}_{\mathrm{x}} \mathrm{H}_{\mathrm{y}}$ in (a) plan-view and (b) side-view. Corresponding FFT's are shown for (c) plan-view and the (e) side-view with characteristic d-spacing values labeled. (d) Background-subtracted XRD spectrum for $\mathrm{g}-\mathrm{CN}_{\mathrm{x}} \mathrm{H}_{\mathrm{y}}$; inset: 2D single-sheet structure model of fullycondensed $\mathrm{g}-\mathrm{C}_{3} \mathrm{~N}_{4}(\mathrm{C}$-atoms=black; $\mathrm{N}$-atoms=blue) with the (100) spacing indicated.
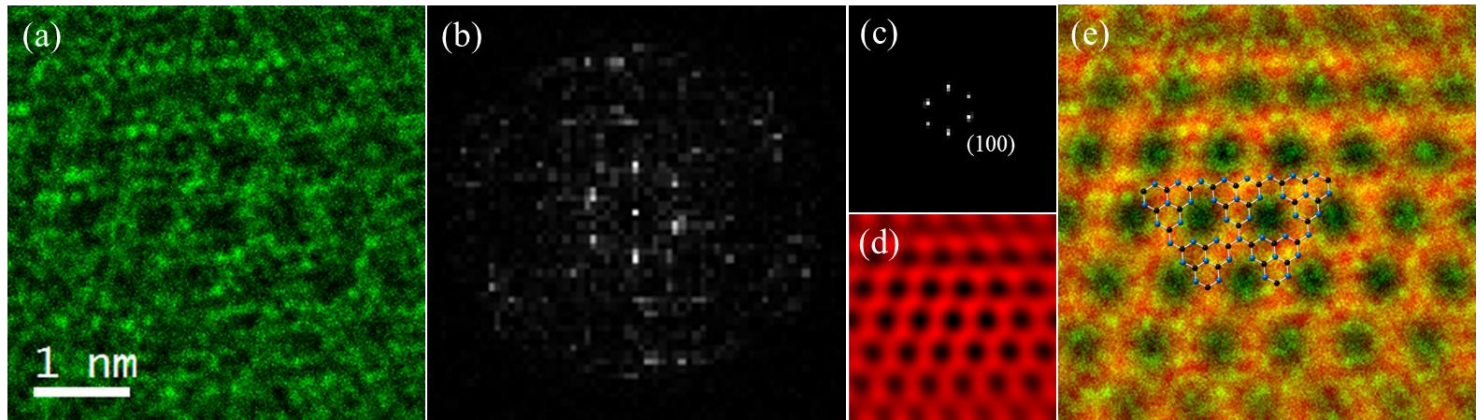

Figure 2. (a) Region of interest from Fig.l(a) with (b) corresponding FFT. (c) FFT after applying a mask to isolate the (100) spots and its corresponding (d) IFFT image. (e) Composite image of the region of interest (green) and the IFFT (red); an overlay of the 2D structure model is also displayed. 